\title{
Group-awareness for Mobile Cooperative Learning
}

\author{
Roc Messeguer, Leandro Navarro and Angelica Reyes \\ Department of Computer Architecture \\ Technical University of Catalonia \\ \{messeguer, leandro,mreyes\}@ac.upc.edu
}

\begin{abstract}
Collaborative learning traditionally relies on the arrangement of space and physical artifacts to mediate group activities. Our claim is that CSCL environments should enhance collaborative learning in a complementary way without introducing additional burden to human participants. This paper present research results on how tracking the location of people could be used to incorporate automatic group awareness in existing and new CSCL applications that support mobile cooperative learning. After presenting the requirements and structure of a middleware to handle automatic group awareness based on location information, two applications are presented. Experiments in a real setting on a jigsaw activity, with students using laptops with WiFi on a university course, confirm the validity of using location technology to automatically derive group structure and the positive effect on the learning results.
\end{abstract}

\section{Introduction}

Cooperative learning [1] is an instruction method based on students working together in small groups to accomplish shared learning goals. Many methodologies of cooperative learning exist, as classified by Slavin [3]. Jigsaw [2, 18] is one characterized by different student tasks: alone, in their formal group and in an expert group, and in the transitions from one task to another where groups change membership and task several times and in parallel during the activity. Detecting these changes, the assembly and disassembly of groups and his membership, is key to understand, support and evaluate the collaborative activity.

In computer-supported collaborative learning (CSCL) [11] the instructor or every student has to explicitly and immediately inform these CSCL applications that support group tasks about who belongs to every group as this changes continuously. The evolving absolute or relative location of every significant element in the workplace (people or artifacts) is a rich source of information to understand the structure and performance of the collaborative task. We intend to use this location information to automatically infer contextual information
[10] (starting with group membership changes) that facilitates CSCL support.

This main contribution of this paper is in exploring ways to support instructors and students involved in collaborative learning activities, and in general in activities requiring work in groups, in detecting the formation, composition and reorganization of groups of students immediately and automatically. This information can be applied during the activity to inform the instructor about the current organization of the classroom (who is currently involved in every group), to support students whenever they are in a given group (CSCL tools can offer an automatic shared context for collaboration to group members), or to collect log information for further evaluation.

Location devices can be used to track the location of students (e.g. bearing identification devices such as active badges or RFID tags) or their computers (e.g. by triangulation of radio signals from laptops or PDA). This information can be periodically processed and including context information (e.g. the location of tables, panels or other elements in the classroom), it may describe in real time the evolution and membership of groups during the collaborative activity. After testing several technologies (RFID, Infrared, Bluetooth and IEEE 802.11 (WiFi)), we selected WiFi as the best option.

Software modules have been defined to collect, filter and store location information about students, and derive group membership information that can be offered as contextual information to applications. An "instant collaboration" application that, based on that group membership information, provide instant messaging and file sharing automatically restricted to these groups, has been prototyped. The design has also been applied to another existing synchronous collaborative application.

These ideas have been evaluated with real students involved in jigsaw activities, using WiFi laptops and several support applications, with positive results.

The remainder of this paper is organized as follows. Section 2 presents related work. Section 3 describes the design issues and architecture for a middleware for dealing with group awareness for mobile cooperative learning. Section 4 describes the environment for evaluation, a real setting with prototype applications and a group of students using laptops with WiFi. 
Section 5 presents the results of experiments firstly in terms of validating the location technology and secondly in terms of the effect on group work and learning. Finally section 6 is a summary of the conclusions, findings of this research and future directions.

\section{Related Work}

There are many proposed middleware for capturing context $[5,9,14]$, but our proposal is restricted to the identification and positioning of group participants as well as information for the dynamic management of groups, not as a human-computer interface.

The Research Unit for Educational Technology at UPC and many other universities have developed pedagogical systems using wireless devices (WiFi laptops or PDA) to improve collaborative learning and knowledge building aiming for providing individual and collaborative "cognitive tools" in-campus and off-campus learning. This work proposes the incorporation of assistive mechanisms to decrease the cognitive load of group participants and reduce the overhead associated to the instructor during the class and providing a $\log$ of activity for later review.

There are several groups doing related research projects in this area. These are: the development of a software abstraction for the available technologies of indoor location $[4,5]$ their use in an educational context $[6,7]$, ubiquitous computing, as an approach to context [8]. In particular, the CatchBob! [6] project by the CRAFT research group which is focused on understanding the influence of space and location awareness on collaboration. In contrast, our proposal is unique as it is oriented to use location information to be automatically incorporated in classroom CSCL applications that are based on group contexts.

$\mathrm{Li}$ at al. in [15] proposes a framework of ubiquitous learning environment, without social interaction between learners. As an innovation we focus on group management support and rules as well as the encounter functions of social interaction communication and collaboration (compare with [16]). Beside, we propose the addition of location to context learn information and the assignation of dynamically rules to learners. In [17], they focus on a context aware environment to support the needs of peer-to-peer collaborative learning. In this paper, we focus on face-to-face cooperative learning with computer support environments.

\section{Design of a group awareness middleware}

The basis of collaborative learning is the willingness of the peers to participate in collaboration in a constructive sense. Arrangements of space and physical artifacts are key resources in the organization of these collaborative activities. Thus, if computer-support is going to be introduced it is desirable that it will be done in a way that adds and not limits the use of the existing spatial resources. The physical environment and the computer based environment are usually separated or mutually updated manually by all or some of the human participants.

Our claim is that a CSCL environment that enhances collaborative learning should possess mechanisms to identify the potential or intention of people to collaborate with each other or to some artifact (a table, a whiteboard, a flip chart, etc). Our experiments determine this potential taking into account the proximity of people. We use different types of positioning technologies; however, all of them require members of the groups to be into a specific area covered by antennas and $\mathrm{WiFi}$ devices.

A context awareness middleware is proposed as a way to abstract from lower level details, to provide a generic mechanism for any application (CSCL or not) to be informed about the current location and the group context of people or other artifacts, and to enable access to the history of locations visited or group arrangements (individually or collectively).

\subsection{The architecture of the context awareness middleware}

Using the current location and context-information of each equipment/person/student, the middleware should create groups and even assign roles for collaborative applications.

We identify three main levels: application, middleware and physical.

The middleware picks up information from the physical level using different mobility and location technologies that provides location information, relative location, physical proximity to artifacts such as tables, etc. It may be possible to use any available and adequate devices based on technologies such as infrared light, ultrasound, and radio (RFID, WiFi, or Bluetooth). Whatever wireless technology is used, our middleware will produce the precise position via a component called "processor". The processor stores the data collected from different sensors and it also processes that information to calculate the estimated position that is going to be sent to the "Manager" modules in real time.

Besides this information, the middleware should also consider previously defined context information (e.g. the characteristics of the classroom: number and location of tables, whiteboards, etc.) and also previously defined rules (e.g. common rules to assign group membership based on 
proximity to a certain artifact, or rules to assign roles based on location or specific gestures in space).

The module in charge of the management of the overlay topology can use current group membership information to create an overlay network private to the group. This may be useful for applications to send messages or discover nodes limited by default to the current group where the node belongs to.

The application level can use context-based positioning to allow participants among a group locate their partners, create a shared information space, for matchmaking between participants of a conference, or to take advantage of location-awareness to position avatars in augmented reality scenarios, etc.

The context-based positioning information can also be used to do research and evaluate the performance of groups, in terms of potential interactions, special relationships, collaborative problem solving, cognition in group, how mobile technology and collaborative activities impacts on group interactions, etc. For instance, a logging application could collect local historic data and send it to the instructor to aggregate data from all students during a certain activity and have a global view of what happened in certain session.

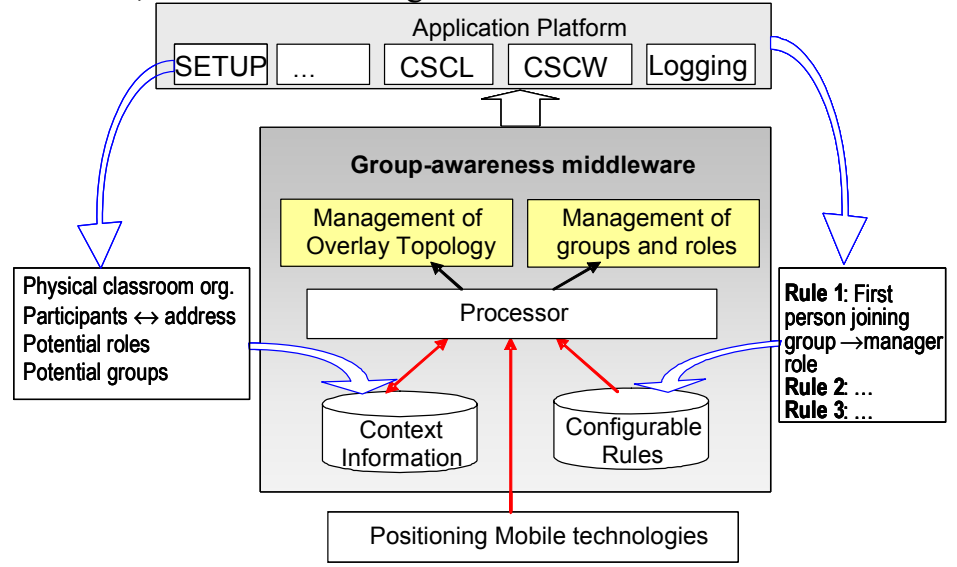

Figure 1. Middleware Components

The figure 1 shows the structure and interactions of the middleware.

The middleware collects positioning information in order to detect when groups are created or disbanded and even to assign a role for each student in the group following certain predefined rules. In this level we are not concerned about the psychological implications produced by belonging to a group and how group interactions affect learning, since the middleware does not consider these effects. The formation of groups is only affected by students' proximity and in some cases by the resources that are nearby to a specific student (e.g. close to a group' table).

Besides positioning information, detecting groups and assigning roles are processes that require a previously defined set of rules the pattern: "If there is a condition evaluated to true then a specific action has to be applied." These rules are a key part of the management module of the middleware, who is responsible for processing the location information and sending the result to the upper application level.

The model consists basically of 4 types of rules:

1. Rules to create groups (e.g. a temporary group is created whenever several people stay together, based on a threshold diameter, in a place for more than a certain threshold period of time)

2. Rules to destroy groups (e.g. whenever membership reach less than 2 or 1 participants for more than certain threshold period of time)

3. Rules to belong to a specific group (e.g. staying closer, based on threshold diameter, to a certain table)

4. Rules to assign a specific role to persons belonging to a group (e.g. the first person arriving to the group becomes the group leader).

\section{Environment for Evaluation}

The approach to evaluate how effective and useful is group awareness using location tracking has been to implement a simplified version of the middleware and applications, and experiment with real users in collaborative learning activities that have been performed already using no computing technology or simply using laptops with no support for collaborative learning. That reference scenario was established as a baseline for comparison, and several experiments were done using two synchronous group-aware CSCL applications developed in the group: Instant Group Collaboration (IGC) and 
Redianet class with and without automatic group awareness respectively.

After several initial debugging experiments to test and refine the location technology, the applications and the learning process including these new features, three instances of the experiments were performed. An analysis of the results is presented at different levels: at the level of determining the location of students and the group dynamics, at the level of effectiveness in supporting the learning and at the level of user satisfaction.

Our intention in this evaluation was to assess how CSCL applications with automatic group awareness that assist group interactions among students result in any measurable improvement in the learning process.

\subsection{Scenario for experiments}

This work has been done at the EPSC campus of UPC, an engineering school built and designed to be based in the collaborative learning and project based learning models. Classrooms are equipped with tables and chairs having collaborative learning in mind. Experiments have been done in a subject where each student has a laptop connected via a WiFi network and they interact with one or more collaborating peers to solve a given problem. These experiments were repeated several times, on slightly different collaborative tasks, using different location technologies and different versions of the middleware. As a result of the experiments we obtained location traces that have been successfully processed to reconstruct the history of people groupings, also questionnaires from the students and the qualifications obtained by students on the topic of that session.

\subsection{Jigsaw activity}

The collaborative learning methodology followed in the experiments performed was a jigsaw. The basic premise of a jigsaw $[2,18]$ is to divide a problem into sections. Home groups are formed, with each team member taking responsibility for one section of the problem in question. Each student receives resources to complete only his part and becomes expert in this. Expert groups are then formed. Students who are responsible for the same section join together and form a new, temporary focus group whose purpose is for the students to master the concepts in their section, and to develop a strategy for teaching what they have learned to the other students in their original collaborative learning group. After the expert groups have completed their work, the home groups, the original collaborative learning groups reassemble. The students then teach one another the sections they have worked on. To ensure individual accountability, the students can be evaluated on all sections of the task.

The stages of the jigsaw activity we followed are:

1. Introduction of the topic (whole class)

2. The teams go over the problem in question and assign a section to each member. (by group)

3. Individual work of each section of the problem (by student)

4. Expert groups work to master the concepts of their section (by group)

5. Home groups work to connects the various section to answer the problem in question (by group)

6. Evaluation (by student and/or by group)

The students and instructors have experience in using this methodology in different courses, only with paper and pencil, and also with laptops just to take notes, but not with additional support for communication and collaboration.

To introduce automatic group awareness two CSCL applications were used: Instant Group Collaboration and Redianet class.

\subsection{Instant Group Collaboration application}

The goal of this application, developed specifically for this research, is to use group information derived from location to automatically offer to each student a shared interaction space, permissions to access documents and disseminate events produced by other people in the same physical group (typically sitting on the same table or in a ring of chairs).

The application offers a shared virtual folder for the group where any member can find all the documents that everyone in the group has made public. As people move from table to table, group membership change and thus the list of files in every group accordingly.

\section{Application}

$-[\square] \times$

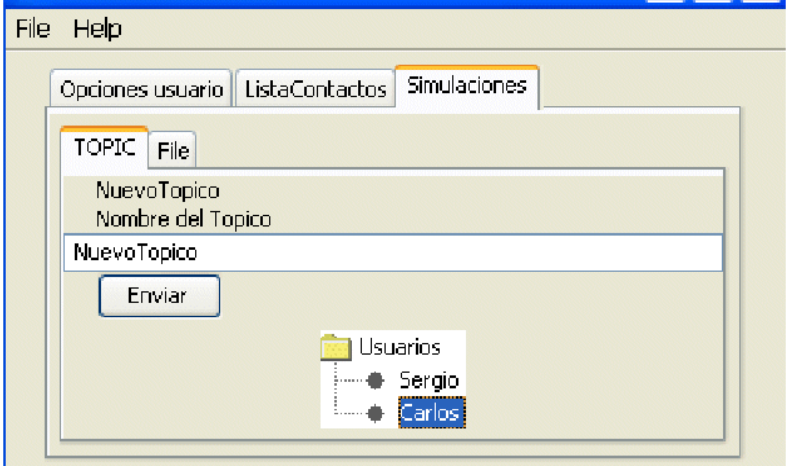

Figure 2. The Instant Group Collaboration offers file and event sharing and dissemination in the context of the current group. 
To identify and locate the students during the experiments we have provided each one with a laptop. The laptops that students carry can be identified by their MAC address, a unique identifier attached to most forms of network interface cards, for example WiFi. The system will detect which students are on each group based on the location of each unique MAC address of the laptops with WiFi they are carrying.

The applications running in each laptop interact with other application instances in a peer-to-peer way based on Pastry [20], a distributed hash table as a routing mechanism for content and for sharing information in a decentralized manner, and Scribe [21] for multicasting information to groups.

In the laboratory classroom students hang around the different tables in order to allow each student's group to share their results and information with the others, using a shared folder created and reorganized automatically as students move.

\subsection{Redianet class application}

Redianet class is a networked program that allows an instructor display its screen and other multimedia contents in real time, observe the screen and act on all the PCs of the class one by one or simultaneously on all PCs and let the students collaborate with its companions by showing the screen, sharing files, acting remotely and exchanging messages on the PCs of the group. This is a commercial application that resulted from a research project initiated in year 2000 by the distributed applications research group of the Technical University of Catalonia (UPC). It was reimplemented and commercialized by Rededia S.L. a spin-off company dedicated to R+D since year 2002 .

The applications running in the instructor computer and on each laptop interact with other application instances using multicast channels. The instructor can broadcast his screen, files, messages, web pages, applications, etc. to all or several PC in the classroom and it can also form groups of students and give them permissions to interact in the same way within the group.

Apart from having different functionality, the main difference with the previous application is in being more oriented to sharing media, and different degree of maturity, the most relevant difference for this research is that here he instructor has the power, but also the additional work, to explicitly organize students in groups. While this may be advantageous in certain situations, in a jigsaw activity this becomes a problem since groups change many times and at the same time during the activity overloading the instructor, trying to cope with the work to inform the application about the frequent a rapid changes of groups.

\section{Experiments}

We validated the dynamic group and role management using experimental measurements (log events), students' questionnaires and comments, classroom non-participant observations and learning assessments.

The experiments were done in a research laboratory where students used laptop computers connected to a WiFi network. This validation experiences was applied to a course in the fourth year of the studies Telecommunications Engineering carried out in the EPSC Campus of Castelldefels at the Technical University of Catalonia. The evaluation was carried out in a class with 27 students.

\subsection{Validation of the Location technology}

Initially we did experiments using RFID tags and Bluetooth technology. The results with these technologies were discouraging given the lack of precision, the high rate of false readings or the difficulty of setting up some components such as the antennas. We then tested with IEEE 802.11 wireless networks adapters (WiFi) used both for network access and for location detection.

We conducted several experiments to measure WiFi based location viability. We evaluated this potential technology (WiFi) for estimating the location of people, computers, and other devices, in indoor locations such as offices, classrooms, laboratories.

The first experiment was done in a research laboratory where students used 5 laptop computers and one PDA all connected to a WiFi network (five access points). In this classroom and their environment, we did some experiments and measurements to estimate the location error of the system. WiFi location using Placelab [4], gave fair results in terms of location. These first experiment shows that we can estimate a user's position in indoor environments with an empirical average positioning error of 1-2 meters (depending upon the characteristics of the environment).

Moreover, we did a second location technology experiment. We did a simplified jigsaw cooperative learning activity. We proposed three readings. The class of 27 students was divided into nine groups of three students. Each member of group read into the laptop a paper, discussed in expert groups and explained to others members of his original group. Every group worked on a different table. Each table was separated by at least 3 to 4 meters from the rest (see figures 3). We manually annotated the group dynamics during the activity and compared to log events of the WiFi location system. The logs collected during the experiment let us reproduce the group activity, indicating on which table every laptop was 
during the experiment, and therefore in this case tracking the location of every student and the groups (one person associated to each laptop, one group per table). The log presents a large number of errors, students present in one table but not detected or detected in another (see table 1). However, when a student saw an error, he just moved his laptop to remove the error and to be associated to the right table/group. This manual mechanism was effective, but these errors can be eliminated with an adequate sampling frequency and adequate filtering. We concluded that a WiFi location system is viable for the location system in a dynamic group management system.

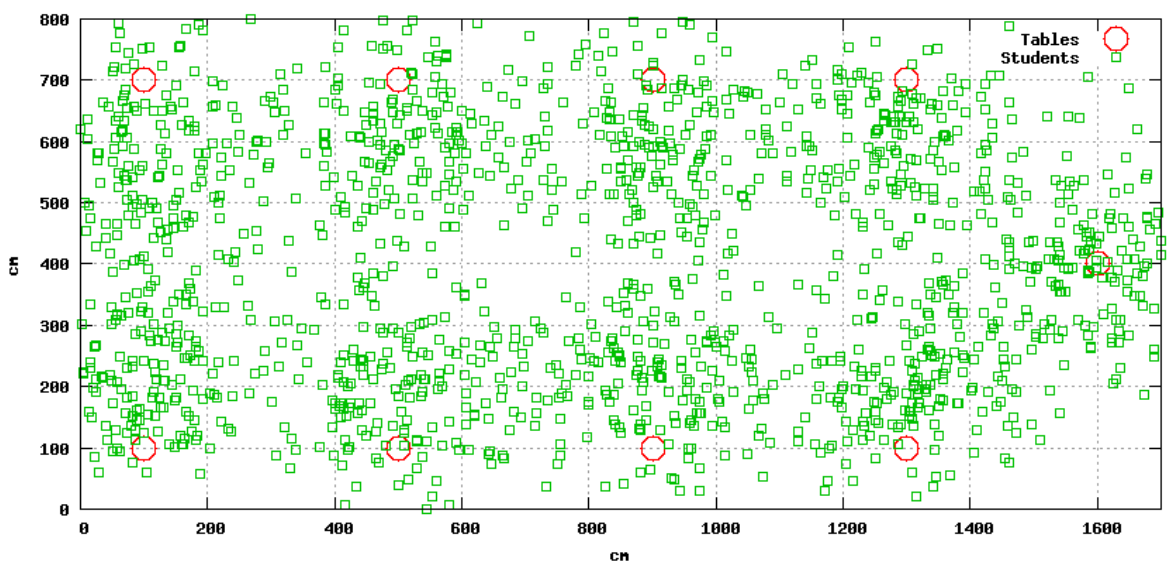

Figure 3. Location of students in the classroom (cm), home group state, (27 students, 30 minutes, 2 samples/min). Tables, with square shape, are represented as a circle giving approximate location.

The hardware was a limiting factor since some common WiFi cards (e.g. Centrino) only can provide location samples every 3-4 minutes which is not adequate for the rate of change in collaborative activities. Instead, we used WiFi cards with the Prism2 chipset obtaining location samples every 30 seconds.

Table 1. Estimation of group for students based on location information with no filtering. Duration of sample: $\mathbf{3 0}$ minutes, 2 samples/minute

\begin{tabular}{llllll}
\hline & $\begin{array}{l}\text { Total } \\
\text { Estimations }\end{array}$ & $\begin{array}{l}\text { No } \\
\text { group }\end{array}$ & $\begin{array}{l}\text { Two } \\
\text { groups }\end{array}$ & $\begin{array}{l}\text { Wrong } \\
\text { group }\end{array}$ & $\begin{array}{l}\text { Error } \\
\%\end{array}$ \\
\hline $\begin{array}{l}\text { Home } \\
\text { group }\end{array}$ & 1621 & 128 & 55 & 144 & $21 \%$ \\
$\begin{array}{l}\text { Expert } \\
\text { group }\end{array}$ & 1621 & 181 & 41 & 86 & $19 \%$ \\
\hline
\end{tabular}

Table 2. Individual qualifications out of 10 (scores from a quiz on the topics covered in the class)

\begin{tabular}{lllll}
\hline & $\begin{array}{l}\text { Location } \\
\text { Group } \\
\text { (IGC) }\end{array}$ & $\begin{array}{l}\text { Redianet } \\
\text { group } \\
\text { (laptops) }\end{array}$ & $\begin{array}{l}\text { Laptop } \\
\text { group } \\
\text { (no app) }\end{array}$ & $\begin{array}{l}\text { Control group } \\
\text { (desktops) }\end{array}$ \\
\hline Average & 8.2 & 7.9 & 7.4 & 8 \\
$\begin{array}{l}\text { Std } \\
\text { Deviation }\end{array}$ & 2.5 & 2.6 & 2.3 & 2.5 \\
\hline
\end{tabular}

Table 3. Group qualifications out of 10 (scores from evaluating the activity group report)

\begin{tabular}{lllll}
\hline & $\begin{array}{l}\text { Location } \\
\text { Group (IGC) }\end{array}$ & $\begin{array}{l}\text { Redianet } \\
\text { group } \\
\text { (laptops) }\end{array}$ & $\begin{array}{l}\text { Laptop } \\
\text { group } \\
\text { (no app) }\end{array}$ & $\begin{array}{l}\text { Control } \\
\text { group } \\
\text { (desktops) }\end{array}$ \\
\hline Average & 9.1 & 8.4 & 7.0 & 6.25 \\
$\begin{array}{l}\text { Std } \\
\text { Deviation }\end{array}$ & 1.9 & 2.6 & 2.3 & 1.2 \\
\hline
\end{tabular}

The IGC application filtered changes of group for just 1 sample: two identical simples were required to change group. This filtering delayed group changes but eliminated most spurious changes (errors).

\subsection{Experiments and evaluation}

We conducted two experiments to measure the viability of determining the group dynamics and role management. Again, we presented the same learning question, using the Jigsaw methodology [18], working on the study of the topic of Content Distribution Networks. In this case, students used the shared virtual folder (IGC) application above described. We will focus on the study of collaboration as sharing information.

As conclusions from the analysis of individual and group scores we can infer:

1. The individually qualification does not depend, or at least not clearly, from the scenario where the activity was performed (see table 2). 
2. The qualifications obtained by the group, do have a clear dependency with the scenario where the activity was performed (see table 3).

That shows how helpful are the WiFi location technologies for quality of the outcome from students groups.

Considering the opinions of students obtained from a quick questionnaire (what worked best and worse), it is clear that the system with a shared group folder contributes to a patent improvement of the group performance reflected in the qualifications obtained by the group.

The great majority of students affirm that either the use of laptops, or the use of Redianet class have been useful in the activity. However, when more details are requested, some responses supporting that statement seem to be less important such as motivation being higher due to the technological novelty. Other responses highlight that these scenarios are more adapted to the activity. A few of them observe an improvement of group work when Redianet class was used, or an improvement in the work in groups and in mobility in the scenario using laptops.

Among negative opinions the duration of the activity appears. Some students claim that either laptops or Redianet class only contributed to spend time by using unknown programs or computers. This was probably due to the lack of experience of the instructor and students who had the additional work of learning to use the tools.

Finally, among the comments from the instructors many technical problems are reported, unrelated to the planning of the activity: load and change of laptop batteries, experience in using the Redianet application, technical problems to access the wireless network, etc. Most of these eventual technical problems can have influence in the results of the experience, even leading to conclusions not fully correct.

A clear observation has been the use of Redianet to exchange information, help, etc. among members of the group. This observation has been reflected in the markings based on group documents.

A final doubt, have been properly adapted the characteristics and planning of the activity for each scenario? Was the jigsaw methodology adequate for this research? The students and instructors had previous experience with jigsaw and this was a rather demanding scenario for evaluating the technology. Based also on the comments from instructors and students the response is positive.

\section{Conclusions}

In this paper we have presented the problem of automating the incorporation of group awareness information to CSCL applications supporting cooperative learning activities such as a jigsaw, without passing this burden to group participants or overloading the instructor. During the activity this results on CSCL applications being able to provide a shared group artifact: a group space for sharing or communication (i.e. an dynamic overlay network for communication within each group), automatically and thus immediately matching the evolving physical grouping of people. In addition, this information can be collected and stored for future (offline) evaluation of the collaborative activity.

A simple application, Instant Group Collaboration, automatically providing group awareness to groups on shared documents and event dissemination has been prototyped. A complex application, Redianet class, providing synchronous support for collaborative learning but with no automated group awareness has been analyzed and evaluated experimentally.

Experimental validation and comparison among different settings has shown that location technologies can be effectively used as a basis for a middleware for the dynamic management of context information in terms of groups and roles. Deriving group membership information from location information based on WiFi networks is technically viable, can be used in classrooms with the adequate dimensions, and can be incorporated in CSCL applications. The use is beneficial for group participants (now free from the burden of informing CSCL applications every time there is any membership change), for CSCL applications (now having current and rather accurate information on group structure). The effect can be perceived in terms of user satisfaction and improvement on the learning outcomes and thus in student's qualifications.

Future work, initiated with Redianet class, includes the detailed definition, implementation, integration and evaluation of this middleware with a complete synchronous CSCL application using context information for the dynamic management of groups and roles, and for the construction of a P2P overlay network adapted (optimized) to the dynamic changes in groups and context in general. Filtering of events require further research, since there is a tradeoff on response time and reliability of group changes, however students were able to easily overcome these errors. Another open issue is using location information to determine the assignment of roles, but this would require additional precision in location and higher sampling frequency.

\section{Acknowledgement}

This work was supported in part by Spanish Government under Contract TIC2002-04258- C03-01. The authors are grateful of Manuel Oneto from Rededia for building and supporting the Redianet application, and 
to Miguel Valero, director of the EPSC campus of UPC, for the technical and educational support for this experience.

\section{References}

[1] Johnson, D.W., Johnson R.T. and Smith K.A.: Cooperative Learning: Increasing College Faculty Instructional Productivity. ASHE-ERIC Higher Education Report No. 4, George Washington University (1991)

[2] Felder, R., Rebecca, B.: Cooperative Learning in Technical Courses: Procedures, Pitfalls, and Payoffs. ERIC Document Reproduction Service ED 377038 (1994)

[3] Slavin, R.E. Cooperative Learning: Theory, Research and Practice (2nd ed.), Allyn \& Bacon, 1995

[4] Place Lab website: http://www.placelab.org/, last visited: April 2006

[5] Hightower, J., Brumitt, B. and Borriello, G. The location stack http://portolano.cs.washington.edu/projects/location/

[6] CatchBob! http://craftsrv1.epfl.ch/research/catchbob/, last visited: April 2006

[7] Active campus http://activecampus.ucsd.edu/, last visited: April 2006

[8] Schilit, B., Adams, N. Want, R. Context-Aware Computing Applications. 1st International Workshop on Mobile Computing Systems and Applications. (1994) 85-90

[9] Dey, A., http://www.cs.cmu.edu/ anind/context.html

[10] Coutaz, J., Crowley, J., Dobson, S., Garla, D.; Context is key. Comunications of the ACM, 48(3) (2005) 49-53

[11] Wasson, B: Computer Supported Collaborative Learning; An Overview. Lecture notes from IVP 482, Spring, University of Bergen, (1998)
[12] Oakley, B., Felder, R.M., Brent, R., Elhajj, I., Turning Student Groups into Effective Teams. J. Student Centered Learning, 2(1), 9-34 (2004).

[13] Doing Cooperative Learning website http://www.wcer.wisc.edu/archive/cl1/CL/doingcl/DCL1.asp, last visited: April 2006

[14] Mostefaoui, S. K., Bouzid, A. T., \& Hirsbrunner, B. (2003). Using Context Information for Service Discovery and Composition. 5th International Conference on Information Integration and Web-based Applications and Services (IIWAS 2003), September 2003

[15] Li, L., Zheng, Y., Ogata, H.; Yano, Y.; A framework of ubiquitous learning environment. The Fourth International Conference on Computer and Information Technology, 2004. CIT '04. Septembe 2004 Page(s):345 - 350

[16] Zhang, G., Jin, Q., Lin, M., A framework of social interaction support for ubiquitous learning. 19th International Conference on Advanced Information Networking and Applications, 2005. AINA 2005. March 2005

[17] Yang, S. J. H.. Context Aware Ubiquitous Learning Environments for Peer-to-Peer Collaborative Learning. Educational Technology \& Society, 9 (1), 188-201. 2006

[18] E. Aronson, N. Blaney, C. Stephan, J. Sikes, M. Snapp, The Jigsaw Classroom, Sage, 1978

[19] Messeguer, R., Diaz de Cerio, L., Navarro, L., Experiencia de aprendizaje cooperativo con una herramienta de colaboración síncrona y ordenadores portátiles. Cuarta Jornada sobre Aprendizaje Cooperativo, GIAC-ICE, UPC, 2004

[20] Rowstron, A., Druschel, P., Pastry: Scalable, distributed object location and routing for large-scale peer-to-peer systems, Middleware'2001, Germany, November 2001.

[21] Rowstron, A., Kermarrec, A.M., Castro, M., Druschel, P., SCRIBE: The design of a large-scale event notification infrastructure, in Networked Group Communication, 2001, pp. $30--43$. 